\title{
TRUSTWORTHY ARTIFICIAL INTELLIGENCE IN EDUCATION: PITFALLS AND PATHWAYS
}

\author{
NATHALIE A. SMUHA*
}

\begin{abstract}
Across the world, Artificial Intelligence (AI) applications are entering all domains of our lives, including the educational environment. This development was further spurred by the COVID-19 pandemic, which rendered many educational institutions dependent on (AIenabled) digital learning tools to continue their activities. While the use of AI systems can lead to numerous benefits, it can also entail ethical risks, which are increasingly appearing on legislators' agendas. Many of these risks are context-specific and increase in significance when vulnerable individuals are involved, asymmetries of power exist, or human rights and democratic values are at stake more generally. Surprisingly, however, regulators have thus far paid only little attention to the specific risks arising in the context of Artificial Intelligence in education (AIED). This paper hence aims to assess the ethical challenges posed by AIED. Its normative framework consists of the seven requirements for Trustworthy AI, as set out in the Ethics Guidelines of the European Commission's High-Level Expert Group on AI. After an overview of the broader context in which these requirements took shape (Section 2), the paper examines each requirement in the educational realm, as well as the pitfalls that should be addressed to enable their realisation (Section 3). Particular attention is given to the special role of education to shape people's minds, and the manner in which this role can be used both to empower and exploit individuals. The paper notes that AIED's main strengths - offering education on a wider scale, through more flexible and individualised learning methods, and through the closer monitoring of students' reception of the materials - are also its main liabilities when left unchecked. Finally, the paper discusses various pathways that policymakers should consider to foster Trustworthy AIED beyond the adoption of guidelines (Section 4), before concluding (Section 5).
\end{abstract}

\section{Keywords}

Artificial Intelligence, Education, Ethics, Trustworthy AI, AIED, AI Governance

FWO Fellow at the KU Leuven Faculty of Law, Department of International and European Law, nathalie.smuha@kuleuven.be. 


\section{Table of contents}

1. INTRODUCTION 3

2. THE LANDSCAPE OF AI ETHICS 4

2.1 The Ethics Guidelines for Trustworthy AI in context 4

$2.2 \quad$ AI Ethics and Education $\quad 6$

3. TRUSTWORTHY AI IN EDUCATION: REQUIREMENTS AND RISKS 8

3.1 Human agency and oversight 8

$\begin{array}{ll}3.2 & \text { Technical robustness and safety } \\ 3.3 & 10\end{array}$

$\begin{array}{lll}3.3 & \text { Privacy and data governance } & 10\end{array}$

$\begin{array}{lll}3.4 & \text { Transparency } & 12\end{array}$

3.5 Diversity, non-discrimination and fairness $\quad 13$

$\begin{array}{lll}3.6 & \text { Societal and environmental wellbeing } & 14\end{array}$

3.7 Accountability 16

3.8 Additional considerations for a Trustworthy AIED assessment 17

4. PATHWAYS TO SECURE TRUSTWORTHY AIED 18

5. CONCLUSIONS 20 


\section{Introduction}

Artificial Intelligence (AI) may well be one of the most hyped technologies of our time, yet its increasing ubiquity in all areas of our lives attests it to be more than a hype. ${ }^{1}$ Being a general purpose technology that can be used to execute tasks more efficiently in a wide range of sectors and domains, AI has the ability to generate substantial economic and societal benefits (European Commission 2018; McKinsey 2018). It is thus no surprise that governments across the globe eagerly adopted AI-strategies to secure sufficient capital, talent and infrastructure to become AI powerhouses - from China's 'Next Generation AI Development Plan' (2017), the UK's 'AI Sector Deal' (2018), the European Union's 'Communication on Artificial Intelligence for Europe' (2018) or the United States' 'Executive Order on Maintaining Leadership in AI' (2019).

The adoption of AI is also growing in the realm of education, where it can build on the accomplishments of educational technology or 'EdTech' more generally. The use of AI-based applications extends to various educational environments - from traditional schools to specialised language centres - aiming to support students, teachers and schools to achieve their objectives more effectively (Southgate et al. 2018; Francesc et al. 2019). This development was further spurred by the COVID-19 pandemic, which led many educational institutions to become dependent on (AI-enabled) digital learning tools to continue their activities amidst restrictive measures.

The development and use of AI, however, also presents certain risks. These risks are not new, yet are increasingly becoming the subject of broader public scrutiny recently. Much thanks to engaged researchers and practitioners, vocal civil society organisations and committed journalists, the technology's adverse impacts are increasingly brought to light (Mittelstadt et al. 2016; O'Neil 2017; Leenes et al. 2017; Yeung 2019; Crawford et al. 2019; Benjamin 2019). Over the past years, certain AI-applications have led to a public outrage when the harm caused thereby - to individuals or societybecame more widely known (Isaak and Hanna 2018; Coughlan 2020; Bedingfield 2020). This triggered a broader discussion on the ethical challenges raised by AI, and on how they should be addressed. Consequently, along with strategies to boost AI's uptake, reflections on how to minimise, prevent and overcome AI's risks also emerged, both at the national and international level.

Moreover, beyond the assessment of AI's risks from a horizontal perspective, calls are increasingly made to analyse and address the technology's ethical challenges also from a vertical or sectorial perspective. Evidently, an erroneous prediction made by an AI system about a movie one may be interested in watching, does not have the same impact as an erroneous prediction about a medical treatment for a cancer patient or the downgrading of students' A-level results (Afifi-Sabet 2020). Since many of AI's risks are context-dependent, a number of initiatives have also started to map these risks in more 'sensitive' domains. This sensitivity can stem, for instance, from the involvement of vulnerable individuals or groups, from a manifestation of asymmetries of information or power, or more generally from the fact that human rights or democratic values can be adversely impacted. Surprisingly, however, despite the unquestionable sensitivity of the educational realm, thus far only little attention has been paid to the specific risks arising in the context of Artificial Intelligence in education (AIED).

$\mathrm{AI}$ is an umbrella term for various technologies demonstrating intelligence, from natural language processing (enabling text analysis and translation) to voice and image recognition (enabling interaction with virtual assistants, or enabling the identification of objects or faces). The most recent successes booked by AI build on increased computing power at lower cost, research advances (particularly in the AI subfield called machine learning), and the growing availability of big data (on which this subfield heavily relies). For a more detailed definition of AI, see e.g. (High-Level Expert Group on AI 2019a). 
In light of the above, this paper aims to assess the ethical challenges posed by the use of AIED. The normative framework of this assessment consists of the seven requirements for Trustworthy AI, as set out in the Ethics Guidelines of the European Commission's High-Level Expert Group on AI. After an overview of the broader context in which the seven requirements for Trustworthy AI took shape (Section 2), the paper examines each requirement in the educational realm, as well as the pitfalls that must be addressed to enable their realisation (Section 3). Particular attention is given to the special role of education to shape people's minds, and the manner in which this role can be used both to empower and exploit individuals. The examination highlights how AIED's main strengths - offering education on a wider scale, through more flexible and individualised learning methods, and through the closer monitoring of students' reception of the materials - also constitute its main liabilities when left unchecked. Finally, the paper discusses various pathways that policymakers should consider to foster Trustworthy AIED beyond the adoption of guidelines (Section 4), before offering some concluding remarks (Section 5).

\section{The landscape of AI Ethics}

\subsection{The Ethics Guidelines for Trustworthy AI in context}

Although the term Artificial Intelligence was established by John McCarthy only in 1956, the philosophy of AI already kick-started a few years earlier in 1950, when Alan Turing published a seminal paper raising the question: "Can Machines Think?" (Turing 1950). Over the following decades, Turing's imagined 'universal computer' became a reality, and information technologies (IT) more generally became incorporated into our everyday lives. Besides questions of theoretical philosophy, the increased use of IT in more practical contexts - and the increased possibilities they created for harm or misuse - also started giving rise to questions of practical philosophy or ethics. Within the domain of ethics, it is the field of applied ethics that deals with the question of what a person ought to do in a specific situation or action domain of action. AI ethics is hence a sub-field of applied ethics, and focuses on the ethical conundrums raised by the development and use of AI systems.

The field of AI ethics counts various ethical theories, each putting forward specific ethical guidance to follow in the context of AI (Lin, Abney, and Bekey 2014). While some believe that traditional ethical theories (for instance based on virtue ethics, deontology or utilitarianism) provide a sufficient ethical framework for AI's development and use, others consider these theories inadequate to tackle the specific issues raised by AI and instead put forward new ethical theories (often heavily drawing on - or containing a mix of - more traditional ones). This difference of opinions immediately clarifies that there is not just 'one' ethics, or 'one' ethics of AI, but that multiple ethical theories around AI co-exist, which, depending on the context or timing, can prescribe different actions or outcomes to pursue. The increased use of AI in society also triggered an increase of attention to AI ethics, resulting in a proliferation of ethical guidelines in this sphere (Jobin, Ienca, and Vayena 2019). The principles contained in these guidelines are typically addressed to developers and deployers of AI systems, who are considered to have an ethical duty - in addition to legal obligations they can have - to secure compliance therewith.

At the European level, the most influential guidance document stems from the European Commission's High-Level Expert Group on AI (HLEG on AI). This independent expert group gathered 52 experts from academia, industry and civil society with different backgrounds, and was inter alia tasked with the drafting of Ethics Guidelines for Trustworthy AI (the Guidelines), aimed at providing guidance for all those developing and deploying AI. Rather than using the notion 'ethical AI', the HLEG on AI framed 
its foundational ambition as 'Trustworthy $A I^{\prime},{ }^{2}$ which it defined as AI that is not only ethical, but also legal and robust (High-Level Expert Group on AI 2019). To be deemed trustworthy, the Guidelines state, AI first and foremost needs to comply with all legal rules that already apply thereto - such as, for instance, privacy or safety legislation (legal $A I$ ). Second, it must respect ethical principles, which may or may not be embedded in legislation (ethical $A I$ ). Third, it needs to be technically robust, ensuring that no unintentional harm occurs - for instance due to inadequate security measures leading to hacks or data leaks (robust AI). These three conditions should be guaranteed throughout the system's lifecycle. The Guidelines hence take a 'values-by-design' approach, in which the need for compliance should already be reflected in the system's design rather than being a mere afterthought.

The Guidelines do not set out the legal obligations that need to be respected in the context of AI, but instead provide guidance on how the ethical and robust character of AI systems can be secured, doing so in three steps - from the most abstract to the most concrete (Smuha 2019). The first step sets out four overarching ethical principles that should be upheld in the context of AI, drawn from human rights law, namely: respect for human autonomy, prevention of harm, fairness and explicability. From these four principles, in a second step, seven requirements for Trustworthy AI are derived, namely, respect for: (1) human agency and oversight, (2) technical robustness and safety, (3) privacy and data governance, (4) transparency, (5) diversity, non-discrimination and fairness, (6) societal and environmental wellbeing and (7) accountability. Finally, the third step provides an assessment list to operationalise each of the seven requirements by raising concrete questions for reflection and implementation. ${ }^{3}$ The seven requirements for Trustworthy AI were blessed by a subsequent Communication of the European Commission, which encouraged all organisations to implement them (European Commission 2019).

While many of these requirements are already partially reflected in existing legislation, no legislation currently covers these requirements in a comprehensive manner. In February 2020, the European Commission hence published a White Paper that identified some legal gaps it intends to tackle (European Commission 2020). The White Paper focuses particularly on AI-applications with a high risk to individuals' safety and fundamental rights, and examines a number of legislative options for a new AI-specific regulation to complement ethical guidance. A formal proposal for legislation is expected in the first quarter of 2021. Simultaneously, the Council of Europe - counting 47 Member States - also announced it would start exploring the feasibility of new binding regulation in this field (Council of Europe 2019).

The ongoing regulatory initiatives of the European Commission and of the Council of Europe are primarily focusing on a horizontal approach, intended to apply to AI systems regardless of the sector in which they are used. Admittedly, the regulatory scope referred to in the Commission's White Paper covering 'high risk' applications - is partially defined by the sensitivity of the sector in which a given

2 It can be pointed out that striving for 'trust' in the context of AI has been deemed controversial, given that trust is an inter-human emotion that cannot be granted to machines (Tschopp 2020; Bryson 2020), and given that the concept of trust entails a delegation of control without any supervision - which is undue in the context of AI. It has also been argued that trust cannot be based on purely voluntary requirements. These interpretations, however, do not correspond to the meaning of Trustworthy AI as put forward here. Rather, it concerns an overarching concept denoting a number of requirements that should be fulfilled by human beings when designing, developing and using AI. The term is hence less important than the requirements it embodies. Moreover, AI-systems do not exist independently but are part of a broader socio-technical environment. Ensuring their trustworthiness hence requires a systemic approach, focusing on the trustworthiness of all actors and processes involved (High-Level Expert Group on AI 2019; Ala-Pietilä and Smuha 2020).

3 This assessment list has been revised after a feedback gathering process, and a revised version was published in July 2020, accessible at: https://ec.europa.eu/digital-single-market/en/news/assessment-list-trustworthyartificial-intelligence-altai-self-assessment. 
AI system is used. The White Paper lists several sectors it considers to be of 'high risk', including health, transport, energy and - in the public sector - asylum, migration, border controls and judiciary, social security and employment services. This list, however, does not include the domain of education.

As noted above, many of the ethical risks raised by the development and use by AI are context-specific. Therefore, any horizontal approach to ethical guidance or regulation should ideally be complemented by a vertical one, recognising the particular risks arising from the domain in which a system is used. While the seven requirements for Trustworthy AI can provide an overarching framework to assess AI's trustworthiness, the concrete implementation thereof should be tailored to the particular concerns raised in a given context. A - so far still limited - number of initiatives focus on a vertical approach. In 2018, the Council of Europe's Commission dealing with the efficiency of justice drafted a Charter on the use of AI in judicial systems and their environment (CEPEJ 2018). Other examples include Singapore's principles to promote fairness, ethics, accountability and transparency when using AI in the financial sector (Monetary Authority of Singapore 2018), and the UK's code of conduct for the NHS dealing with the use of AI in healthcare (UK Department of Health and Social Care 2019). Moreover, a number of initiatives have started to map the impact of AI on children (UNICEF 2020).

As regards the educational sector, several reports describe the benefits and risks arising from the use of AI in this field (Francesc et al. 2019; Vincent-Lancrin 2020). The Beijing Consensus on Artificial Intelligence and Education, adopted by UNESCO Member States in May 2019, also contains a section on the need to ensure the 'ethical, transparent and auditable use of education data and algorithms', thus acknowledging the risks AIED might generate (UNESCO 2019). Nevertheless, tailored ethical guidance for the development and use of AIED remains sparse (note, however, Aiken and Epstein 2000). AIED does not operate in a legal vacuum and numerous legal rules already apply thereto. Yet AIED developers, educational institutions, and impacted stakeholders have little guidance at their disposal to assess the specific ethical pitfalls that should be considered when developing or using AIED.

\subsection{AI Ethics and Education}

Within the domain of education, AI techniques can be used for many different applications and aims (Andrea Kulkarni 2019). Examples include AI-applications that teach content, such as languagetraining apps, AI-systems that suggest personalised learning trajectories based on student profiles; systems that correct exams through natural language processing or that evaluate oral presentations through video analysis; chatbots that answer student questions; facial recognition applications used for exam proctoring or to monitor students' class attendance; facial analysis applications to assess students' understanding of the materials or their attention span and AI-based evaluation systems that predict and assess students' grades and progress more generally. Besides applications that are student-oriented, AIED applications can also be tailored to teachers, or even be used to evaluate the performance of teachers, for instance through value-added-models (Amrein-Beardsley 2014).

While all these applications belong to the sphere of education, their purposes and ways of functioning are very different. These applications do not all have the same level of (proven) effectiveness, they do not all collect the same amount or type of data, and they do not all have the same level of intrusiveness into a student's or teacher's life. Accordingly, not all AIED applications raise particular ethical concerns. AIED can hence not be reduced to one type of application. The ethical assessment of educational AI applications thus requires an approach that is tailored to the specific use case.

At the same time, within the broader sphere of education, some commonalities with regard to AI's ethical risks can be identified. The most important common ethical concern to consider in AIED context is whether the application entails the direct involvement of vulnerable individuals. These are typically 
children but, in some situations, can also comprise adults who, for instance, are at risk of exclusion (Prinsloo and Slade 2016). In addition, educational settings are typically characterised by asymmetries of power and of information. These asymmetries tend to manifest themselves at three different levels.

First, asymmetries of power and information are inherent in virtually all student-teacher relationships, regardless of the student's age. By definition, teachers have more knowledge on certain topics than their students, whom they are also meant to evaluate. This asymmetry is exacerbated when another vulnerability is at stake, such as a young age, minority background or (learning) disability. Second, these asymmetries also exist between teachers and schools, as teachers typically depend upon schools for their employment and, by extension, for their livelihood. As a consequence, they may find themselves in a difficult position when facing concerns about certain AI applications in their classrooms - whether these concerns arise from the application's ethical risks or lack of effectiveness - especially where their employer heavily invested in the technology and strongly supports its use. Finally, asymmetry of information is often also a factor in the relationship between educational institutions and AI-developers that commercialise AIED products. Schools - and by extension teachers and students typically lack the expertise to accurately understand how educational AI systems work, which data they collect and process, how they arrive at their decisions or recommendations, and what the consequences of the use of these systems might be, including potential adverse effects.

As a consequence, when assessing AIED's ethical challenges, it is essential to look beyond the studentteacher relationship. All stakeholders should be considered to identify where vulnerabilities or asymmetries - in whichever form - might exist and could potentially lead to an exacerbation of AI's risks (Aiken and Epstein 2000). To identify and counter these risks, the seven requirements listed in the abovementioned Guidelines for Trustworthy AI might be a helpful starting point. Whilst not claiming to provide an exhaustive overview of potential concerns, these Guidelines can stimulate a reflection not only on what the ethical challenges of a specific AIED application may be, but also how these challenges could be mitigated. Such reflection should occur ex ante, prior to the system's use - and ideally also prior to its development.

While all AIED stakeholders have their own part to play in securing the requirements for Trustworthy AI, the Guidelines particularly highlight the role of three sets of stakeholders, which can be translated into the educational domain as follows:

(1) Developers of AIED applications (often but not always commercial companies, as well as researchers in the field): they should implement and apply the requirements in their design and development processes;

(2) Deployers of AIED applications (typically schools, whether primary, secondary, tertiary or non-traditional educational institutions - but in some instances, this can also include public entities): they should ensure that the systems they use, and the products and services they offer, meet the requirements;

(3) End-users (typically students) and all other parties affected by AIED (including parents, but also society at large): they should be informed about these requirements and be able to request and enforce accountability.

Arguably, to the extent the educational sector is also a regulated one - or at least one in which the government typically plays a considerable role - a fourth stakeholder and responsibility should be included:

(4) Regulators (whether at local, national or international level): they should ensure that developers and deployers of AIED implement the requirements, and that those affected by AIED - either directly or indirectly - are safeguarded from any adverse impact thereof. 
Attention to AIED-related risks has so far mainly focused on privacy concerns, particularly in light of AI-enabled processing of students' personal data, for instance with the aim of fostering personalised or adaptive learning applications. These concerns also played an important role in the fall of US-based edtech company InBloom even before it was properly launched, which in turn triggered a backlash against AI applications in schools more globally (Bulger, McCormick, and Pitcan 2017). The breach of privacy was also at stake in August 2019 in Europe, when a Swedish school that introduced AI-based facial recognition to monitor student attendance received a substantial fine for breaching EU data protection law (Hanselaer 2019). While privacy risks are certainly important to consider, AIED can raise other risks too, as was for instance demonstrated by the UK's downgrading A-level results system (Coughlan 2020; Afifi-Sabet 2020). It is therefore essential that the ethical assessment of AIED is sufficiently comprehensive and covers all seven requirements for Trustworthy AI, of which privacy is only one. In what follows, each of these requirements is discussed in the context of education.

\section{Trustworthy AI in education: requirements and risks}

\subsection{Human agency and oversight}

By automating certain tasks and decisions that are normally undertaken by human beings, AI by definition has an impact on human agency - yet this is not necessarily problematic. After all, the objective of AI systems is to ease people's lives by taking over some of their work. AI systems can, for instance, execute tasks that are deemed too dull or repetitive to be of human interest (such as replying to 200 student emails asking the same basic questions). They can help carrying out tasks on a much larger scale (such as correcting 2000 identical student exams). And in some instances, they can carry out tasks with more precision and effectiveness than humans could (such as providing personalised curricula for 2000 students based on individual preferences and abilities in a short time span). As long as human beings can meaningfully decide when and under what conditions decisions are delegated to an AI-system, human agency is not only preserved, but can even be empowered. At the same time, the delegation of certain tasks to AI-systems also carries some risks, especially when the delegated decisions can have a direct - and potentially negative - impact on individuals. Importantly, the choice to delegate decisions to AI-systems is a human choice and hence falls under human responsibility.

Safeguarding human agency hence means that all those who are involved in the decision to deploy or use an educational AI-system - be it schools, teachers, students and/or their parents - are enabled to make an informed and autonomous decision. For this purpose, they first need the knowledge to understand how a particular AI-application works, and should have the possibility to challenge the decisions it makes where needed. Whilst it can - and should - be expected from the schools that choose to deploy AI to inform and educate themselves about the capacities and limitations of the system prior to its use, for (end-)users (such as students and teachers) this information should be proactively provided. This information will then enable individuals to exercise their human agency and to assess the appropriateness of the task delegation and the potential risks to be considered.

Human agency can also be hampered in more subtle ways. AI can, for instance, be used to influence student behaviour in a manner that is more difficult to detect, through covert manipulation or deception, or by providing targeted (dis)information. Of course, all educational activities aim to shape human minds in a certain sense, whether by providing new skills and knowledge or by providing additional or new perspectives. Yet this powerful ability of education to shape minds - especially when it concerns young minds that are typically more malleable - can also be used to foster behaviour that might benefit those developing and deploying the technology whilst not necessarily be in the learners' interest. More far-reaching, it can also be used to teach certain beliefs that run counter to the values of human rights, 
democracy and the rule of law, and that foster instead more oppressive or totalitarian goals. Unfortunately, examples of the instrumentalisation of education towards such aims are not only historical (Kunzer 1938; Gallo 2015), but are also found today (Zenz 2019; Raza 2019). And while the use of education for (political) indoctrination does not necessitate AI, the availability of an AI-powered educational infrastructure offers significant advantages to achieve such aim.

Technologies, including those enabled by AI, are inevitably moulded by the knowledge, norms, culture, power relationships as well as (unconscious) biases and prejudices of their creators, and hence valueladen, even if unintentional and barely perceivable (van de Poel and Kroes 2014). In a bleak scenario, this feature can hence be used to purposefully mould AIED so as to influence, nudge and even manipulate students in a more effective manner, whether openly or covertly. Moreover, potential resistance to the curriculum's changes by human teachers could be more easily circumvented or overridden, as the role of human intermediaries might become smaller. In other words, one of the main strengths of AI-based education - its ability to offer education on a much wider scale, at a faster pace, through more flexible methods, and in a way that allows for the closer monitoring of students' understanding and reception of the materials - is in this sense also one of its biggest liabilities. Evidently, the extent of this risk is directly correlated with the role that AIED plays in the classroom. Most democratic societies already have some regulatory safeguards in place in this regard, for instance by regulating the mandatory content that each student should be taught. Moreover, this risk is less present when AIED is used outside of traditional educational institutions rather than in primary and secondary schools. Yet the potential for (covert) AI-based nudging to influence students in more subtle ways - akin to the use of such techniques on social media - remains relevant.

One of the most important ways to counter the use of AI that may (un)intentionally hamper human agency or cause other adverse impacts, is the establishment of strong human oversight mechanisms. The Guidelines mention three particular types of oversight that can help achieve this, which - depending on the use case - should be used complementarily. The first is a human-in-command (HIC) approach, whereby the overall activity of the educational AI-system is overseen, as well as the broader implications it has from an ethical, societal, legal or economic perspective. This also encompasses responsibility for a decision on whether or not to use an AI-system in a given situation in the first place, and - if so - how the system should be used. It is, for instance, one thing for a school to decide, together with its teachers, that an AI-based value-added model will be used to help provide insights into teachers' performance. It is another thing for a school to decide that, based solely on the evaluations of such model, teachers may get fired (O’Neil 2017).

The second oversight approach mentioned in the Guidelines is a human-on-the-loop (HOTL) approach. This approach not only enables human intervention during the design phase of the AI-system, but also ensures that - once the system is in operation - its use is closely monitored. The third is a human-inthe-loop (HITL) approach, whereby human intervention is made possible in every decision cycle of the AI-system. While the latter approach would be too demanding for an AI-system that only presents low or no tangible risks, more continuous human intervention may nevertheless be a necessary safeguard for AI-applications that can significantly impact students' or teacher's lives. It is the responsibility of those involved - including the organisations that develop the system and the entities that decide to use it - to assess, on a case-by-case basis, which oversight mechanism is needed to ensure that the system meets the requirements throughout its lifecycle. This assessment also necessitates prior reflection on the various rights and interests at stake when implementing the system. In this regard, the UN Convention on the Rights of the Child can be recalled in particular, which lists some important rights to considered whenever children are involved. In addition, ongoing initiatives that aim to map the impact of AI specifically on children (UNICEF 2020) can also provide a helpful framework. 


\subsection{Technical robustness and safety}

Like all software systems, AI is only as good as its technical infrastructure. A lack of attention to the system's robustness can still lead to numerous types of harm, which developers and deployers of AI should strive to avoid. Especially where AI relies on (personal) data of students, adequate security measures must be taken to ensure that no data leaks can occur. AI systems should be protected against technical vulnerabilities by making them resilient to attacks on their software (comprising their data and model) or on their hardware. Moreover, whenever AI-systems are embedded in - or linked to hardware, such as robotics, additional caution is also needed for physical damage that can potentially ensue, whether through the exposure of the system to use situations that were not initially foreseen or through the system's intentional corruption by malicious actors. These risks must be assessed and addressed before AI-applications are introduced in educational settings, to secure the physical and mental integrity of all those who might be impacted.

Technical robustness also implies that the system can make correct or accurate judgments. Depending on the use case, inaccurate decisions, predictions or recommendations can have negative consequences for those involved. Examples in the educational context range from an AI-system that recommends the wrong content based on an inaccurate assessment of the student's ability, that wrongly evaluates students or teachers, or that provides them with inaccurate feedback. Accuracy problems are especially relevant for AI-systems that claim to have the ability to read cognitive or emotional states, besides posing significant risks to privacy (Lieberman 2018). This type of technology is increasingly used in other sectors, for instance to analyse video-interviews of prospective job candidates (Harwell 2019), and has started to be deployed in classrooms too. Importantly, however, numerous researchers have pointed to the inherent flaws of such technologies and the unscientific grounding thereof (Bjørnsten and Zacher Sørensen 2017; Barrett et al. 2019). In addition to being generally inaccurate, those systems are disproportionately inaccurate when it concerns students with different profiles, such as those having a disability or coming from a different cultural background than the profiles based on which the system was trained (Crawford et al. 2019).

Finally, the Guidelines also list reliability and reproducibility as necessary attributes under the umbrella of AI's technical robustness. Reliability denotes the need for AI-systems to work properly within a range of inputs and situations, and to effectively carry out the tasks that it claims to do. This means the system must be able to perform these tasks not only within the conditions of the (digital) lab in which it was developed and tested, but also in real school settings. Reproducibility demands that AI-systems yield the same outcome when used under the same conditions, and are hence able to reproduce their behaviour. Whilst the responsibility to verify and secure the technical robustness of AIED rests primarily with the companies developing it, schools and other deployers of AIED must ensure that the systems they use meet these criteria, and - in case of harm - should not deflect their responsibility on the developers.

\subsection{Privacy and data governance}

The importance of the right to privacy and the protection of personal data - two distinct fundamental rights under EU law - is not new in ed-tech debates. Yet the emergence of AIED - and in particular applications based on learning-techniques - only raised the privacy stakes to a higher level, in view of the large amount of data they require. The role of data is not only essential during the system's training phase, but also once it is used. Moreover, data generated in the course of the system's operation can also be re-used as new input. Developers and deployers of AIED applications often operate under the premise that the collection and analysis of as much as possible relevant data about students and their 
(historical and actual) performance can help assess their abilities and provide tailored tools for enhancement (Rouhiainen 2019). While the analysis of student data can be used for many beneficial applications, it also comes with an important responsibility, as it can be used in ways that are not in the students' interests or even run counter thereto.

In order to build a personalised learning trajectory based on individual student profiles, for instance, data is typically collected on their grades, acquired knowledge and learning capacities, but potentially also of their character traits, preferences, health status, family situation, and various other aspects that might be of relevance to map their abilities and set out their learning path. Even if not all these data points are explicitly collected, inferences of a personal nature can still be made - including from information as limited as the movements of a computer mouse (Hibbeln et al. 2017). Companies developing AIED typically monetise such data primarily through their products. Yet questions around the ownership of both the collected data and the outcomes of the analyses based on that data are often undefined, which opens up further risks (Herold 2018). The collected or inferred data could for instance be sold to third parties (including online marketers, insurers or employers) who develop their own AI products and who might use the acquired information for their own purposes.

As raised above, students are not only in a vulnerable position because of their young age, but also because of their dependency on the school and teachers. They are hence not always in a position to challenge the collection of their data. Furthermore, due to the inherent power imbalance in educational contexts, it can even be questioned whether consent can ever be used as a valid legal basis to process student data. To be valid, consent needs to be freely given. However, recital 43 of the EU General Data Protection Regulation specifies that "consent should not provide a valid legal ground for the processing of personal data in a specific case where there is a clear imbalance between the data subject and the controller". In this regard, the crucial role of parents or guardians who, depending on the students' age, can be required to give consent on their behalf should also be considered, not least because in some situations, tensions may arise between the interests of the child and the interest of the parents.

The question can also be raised whether a genuine opportunity to opt out of the technology's use still exists once it adopted by the school. Opting-out might become de facto impossible either because of the pressure exerted upon students and parents to conform to the will of the majority who might believe the application's benefits outweigh the risks, or because the physical infrastructure is put in place and does not technically allow for deviations of the system's use. As noted above, data can also be collected on teachers (for instance to evaluate their teaching abilities (O'Neil 2017)), which - light of the specific employer-employee relationship - can raise the same question of power imbalance and validity of consent.

Assuming that a valid legal basis for the collection of personal data exists, developers and deployers of AIED must protect such data throughout the life cycle of the system. Moreover, this protection should also extend to all the inferred data or the output generated by the system based on the initially collected data. It can be noted that not all AI-systems necessarily rely on personal data to operate. However, AIsystems that do not process personal data can potentially have a (negative) impact on individuals and groups - an example thereof being an AI-application that helps correcting anonymised exams but fails to do so accurately and thus erroneously fails a student. Accordingly, even for AIED applications where no or only little personal data is processed, their impact on the private lives of those involved must be assessed.

The protection of privacy and personal data are not only ethical but also legal requirements, which by now are well established in the educational realm. However, ensuring the ethical handling of data covers more than merely meeting legal standards. Trustworthy AIED also requires that mechanisms for good data governance are established. The quality of the datasets used must be assessed and held to high 
standards (Hasselbalch 2019). Any AI-system will, after all, only be as good as the data it is fed. Therefore, prior to training an AIED application, developers must take measures to eliminate inaccuracies as well as unjust biases from the datasets they use. Securing data integrity throughout the system's lifecycle is essential, as the inclusion of erroneous data into the system can change its behaviour in a damaging way. Sound data governance also requires protocols to govern data-access, outlining who can access information and under which conditions. While AIED developers have the primary task of testing and documenting the steps taken to secure privacy and good data governance, as already stressed above, organisations deploying AIED have an equally important responsibility to ensure that the applications they purchase meet these requirements.

\subsection{Transparency}

To secure Trustworthy AIED, the various elements constituting the AI-application need to be made transparent, including the type of data, the system's model, and the business model for which it is used. Transparency hence functions not just as an ethical value in itself, but rather as a meta-value that sheds light on whether and how all other requirements for Trustworthy AI are respected. In its Guidelines, the HLEG on AI lists three sub-requirements that need to be fulfilled in order to enable this meta-value: traceability, explainability and communication.

Traceability pertains to rendering visible the traces of how a particular decision taken by an AI-system was reached. This requires the availability of documentation that logs which data was gathered and under what conditions (during the training, testing phase and use phase of the application), the choices made for data labelling and categorisation, the selection of the algorithm, and the criteria or variables selected for the algorithm's optimisation. The decisions made by the AI-system (and how these decisions might change under different conditions - for instance in case of slightly different data sets or models) should also be documented, as this not only increases transparency, but also allows to determine why a specific decision was erroneous and how such errors can be prevented in the future. While documenting these elements might sound resource-intensive, various (technological) tools can be used to facilitate this task. Moreover, documenting these elements not only forces developers of AIED to be explicit about the design choices they make and hence to rationally reflect thereupon, but it also enables the auditability of the system by third parties (for instance independent auditors or governmental agencies). Of course, private companies that develop AI-systems are entitled to intellectual property rights and to protect potential trade secrets associated with their systems. However, traceability requirements need not hinder those rights and can coexist therewith.

Especially when the system is used by public entities and has the ability to affect the interests of a large number of students or teachers, documentation that enables the auditability of a system should be provided not only ex post (after the system has been implemented) but also ex ante (prior to the system's use). The example of the algorithm used by the UK's Office of Qualifications and Examinations Regulation (Ofqual) during the COVID-19 pandemic to predict students' A-level grades in the absence of traditional exams illustrate this (Afifi-Sabet 2020; Bedingfield 2020). If Ofqual would have made information about the algorithm and its intended purpose publicly available prior to its implementation, stakeholders and independent experts would have been able to assess the algorithm's robustness and its intended scope of application. On that basis, they would have been able to suggest adaptations to the model or to the manner in which Ofqual envisaged to deploy it. In turn, this would have enabled both sounder and more legitimised policy-making. While this reasoning holds especially true when the system is applied to a country's entire student population, it also holds true for AI-systems that are used at a smaller scale and are able to significantly affect the interests of only a few (Han 2020). 
Enabling an AI-system's traceability also helps to fulfil the requirement of explainability. Explainability demands not the documentation, but the explanation of the (technical) processes of an AI-system. This also comprises an explanation of the human decisions relating to the system, such as for instance in which modality the system is used within the organisation, the rationale for the design choices made and in which area of application it is used. For AI-systems used in learning platforms, an explanation should for instance be given as to why a specific learning trajectory was recommended rather than another, or in the context of AI-enabled grading, it should be explained based on which criteria a specific grade was attributed to a student. Whenever the use of an AI-system can have a significant impact on a person's life, such explanation should always be given proactively, allowing the person to understand how the impacting decision came about. This explanation needs to be provided in a language that is adapted to the knowledge of the person concerned. In other words: this explanation will not be the same when given to a child, an adult student or parent, or an expert. Without such explanation, the individual concerned will not be able to challenge the decision, and will also not be able to seek correction or redress in case it was erroneous or biased.

Lastly, the requirement of transparency necessitates appropriate communication by developers and deployers of AI-systems. This means that, first of all, those interacting with - or subject to - the AIsystem need to be aware that they are not interacting with a human being. Such misunderstanding can particularly occur in the context of chatbots that can be used to communicate with students. The option not to interact with an AI-system but instead to rely on a human interlocutor should also be considered, hence enabling a right to opt out. In addition, the capabilities and limitations of the AIED applications should be communicated both to the deployers of the AI system (e.g. the school that acquires the application) and to the users of the system (e.g. the teachers and students interacting therewith). Such communication should occur in a manner that is adapted to the intended audience. For this purpose, a mapping should be made of the various stakeholders involved with the AIED application in question covering schools, teachers, students, parents and others - and their various needs for information about the system's functioning within its overall environment. Adequate communication is particularly important to avoid over-expectations about the system's capabilities.

\subsection{Diversity, non-discrimination and fairness}

Bias - or the inclination of prejudice towards or against a person, group, object or position - is an inherent part of society and of how human beings operate (Steinbock 1978; Banaji, Hardin, and Rothman 1993; Begby 2013). As subjective individuals, we are unable to grasp anything we encounter in a purely objective manner, but always do so through the filter of our personal knowledge and experience, which in turn constitutes a reflection of our upbringing, education, cultural exposure, personality and numerous other traits. In this broad sense, bias is not necessarily bad. Yet, under certain conditions, bias can be unfair or lead to discrimination. AI-systems are developed by human beings, and fed with data collected and shaped by human beings. It is hence no surprise that their decisions also reflect the individual and societal biases of their creators and users - for better or worse (Silberg and Manyika 2019; Ntoutsi et al. 2020). However, because these decisions arise not from a human being but from a machine - which is considered as an object and hence, mistakenly, as 'objective' - and because bias in machines is often less perceptible, this risk is crucial to address (Benjamin 2019; Crawford et al. 2019).

Unfair bias can manifest itself in numerous ways in AIED. Often, biased predictions or decisions are the result of a biased data-set on which the algorithm is trained. Consider in this context the use of AIbased speech recognition systems to evaluate a student's (language) skills or knowledge. If these systems are trained on a data-set that contains primarily male and accent-less voice recordings, they 
will not be suitable to analyse the voices of a wide range of users, and will be negatively biased against women, migrants with accents or people with speaking disabilities (Johar 2016; Guo et al. 2019; Koenecke et al. 2020). Similarly, facial recognition technology is often predominantly trained on images of white males, and therefore less accurate as regards the faces of women or people of colour (Crawford et al. 2019). Hence, if an educational institution for instance resorts to exam proctoring based on a biased facial recognition system, certain students will be unfairly disadvantaged. This is precisely what happened when such proctoring application was used during the pandemic, and students of colour saw themselves forced to project a white light on their face during the exam so as to ensure the application would continue to recognise them (Johnson 2020; ACLU 2020).

Bias can also emanate from a biased algorithmic model, or it can arise because a system is trained in a given context, but thereafter used in a context that was not initially foreseen. It can also emerge at a later stage, during the system's use phase for instance, if the system learns from and adapts itself to biased interactions with users. Finally, the AI-system can also be deployed in a discriminating fashion. This can be illustrated by revisiting the previously raised example of Ofqual's A-level algorithm. Ofqual's choice to only use the algorithm for schools with larger classes posed an unfair disadvantage for students in larger - often public rather than private - schools (Bedingfield 2020).

Problematically, the unfair biases set out above are often difficult to detect due to their unintentionality and due to the non-transparency of the algorithmic decision-making process and of the human decisions relating to the algorithm's use. Moreover, these biased systems tend to exacerbate existing inequalities by disproportionally affecting those who are already disadvantaged. To counteract these risks, oversight processes should be put in place from the system's design phase onwards. A clear analysis of the system's purpose, operational sphere, constraints and requirements should be made, as well as an assessment of the instances in which unfair bias can emerge, for instance the data collection or selection, the codification of rules, the development of the algorithmic model or the deployment by (end)users. Diversity in the teams that design and train AI systems - not only in terms of culture and gender, but also in terms of disciplines and areas of expertise - can help limit these risks, as it fosters a diversity of perspectives that can be embedded in the system.

In addition, AIED developers must ensure sufficient attention to user-centricity, allowing users from diverse backgrounds and with diverse traits and abilities to have access to the AI-application, without being disadvantaged thereby. The standards for universal design as set out by the United Nations Convention on the Rights of Persons with Disabilities can be a guiding framework in this regard, so that the widest possible range of users can benefit from the technology - hence truly democratising education (United Nations 2006). Finally, the Guidelines also suggest the organisation of stakeholder consultations, so that those directly or indirectly affected by the AI-systems can contribute to (the decisions on) its design and use. The solicitation of feedback from those involved - be it schools, teachers, students or parents - is beneficial not only to detect potential errors or bias in the system, but also to further improve the utility and user-friendliness of the application.

\subsection{Societal and environmental wellbeing}

Besides assessing the immediate impact of AI-applications in the area of education, an assessment should ideally also be made of the wider effects that the development and use of such applications can have.

An indirect but critical effect of the use of $\mathrm{AI}$ in an educational context concerns the social impact on students, for instance in light of their interaction with machines rather than human beings. Depending on the specific purpose of the system and role it plays in the classroom, AIED might change students' 
conception of social agency, alter the social relationship between student and teacher (which typically goes much beyond a mere pedagogical role), as well as impacting students' sense of engagement. Only little research has been conducted on the long-term effects of increased or ubiquitous machine interaction in learning environments, and the impact on students' social skills and mental wellbeing. In addition, the use of AI-enabled camera's or monitoring systems might also lead to a chilling effect, as students (but also teachers) might feel observed, potentially leading to changes in their behaviour and hampering their ability to act authentically. The severity of this risk is directly related to the invasiveness of the AI application in students' curriculum, and hence strongly use case-dependent. However, as already mentioned above, both in explicit and implicit ways, AI applications can change not only the way we behave but also the way in which we think, and the social consequences thereof need better mapping.

In addition, while some are cautioning for the potential emergence of intellectual laziness (for instance due to constant interaction with machines that are 'superior' in knowledge), others have raised concerns about how the limitations of these systems shape our learning environments and needs rather than the other way around (Yujie 2019). The data that can be collected on students' reports, grades and progress to feed AI systems, are most useful to track and assess abilities and skills that can be measured by numbers and performance metrics. The more standardised these metrics are - and the tests and surveys that aim to assess them - the easier for the system to compare and analyse. There is thus a risk that - as the usefulness of AI systems is strongest when measuring capabilities in a standardised way - schools will reinforce the use of standardised tests to further enhance the systems' usefulness. However, skills that are easily measurable are also precisely the skills that will be increasingly automated and hence less critical for students to excel in (Bulger 2016). It is therefore crucial that sufficient attention is given to the development of other capacities that are less easily tracked and measured, including soft skills such as teamwork abilities, communication proficiency, empathy and emotional intelligence, as well as critical and creative thinking - even if AIED may not be the best tool to foster this.

A comprehensive impact-assessment of the use of AI-systems in education also entails an examination of the resources they use throughout their lifecycle and their impact on the environment. Many AIapplications require the processing and storing of huge amounts of data, which turns them into notorious energy consumers and undermines their sustainability (Schwartz et al. 2019; Strubell, Ganesh, and McCallum 2019; Hao 2019). While AI can be used to help fostering Sustainable Development Goals (Vinuesa et al. 2020) - including quality education - in order to be truly sustainable, its impact on the planet and on future generations must be born in mind. Therefore, regardless of whether an AI-system is used in the sector of education or in another domain, its environmental footprint - and that of its entire value chain - should be minimised.

Finally, AI's impact on society and democracy can also be considered more broadly. Scandals like Cambridge Analytica have already demonstrated how AI can be used to influence the democratic decision-making process by influencing what type of content people see, thereby shaping their thoughts and emotions in very subtle and almost imperceptible ways (Stark 2018; Isaak and Hanna 2018; Brkan 2019). It is however underexplored what a similar use of AI could mean in the educational realm, the task of which is essentially to shape the democratic citizens and voters of tomorrow. As noted above, while the use of education as a weapon to shield totalitarian and repressive regimes from criticism is not new, AI-enabled educational infrastructure facilitates this risk, not only in terms of scale and timing, but also in terms of subtleness, thus limiting the awareness of the problematic manipulation. It was already stressed that bias is an inherent aspect of human society. Therefore, every educational intervention - whether it concerns an account of history, geography, literature, language, art, economy or biology - will reflect a certain bias by the teacher, whether a human or a machine. In liberal 
democracies, this bias will in principle be coloured by values such as political, religious and scientific freedom, human dignity and equality, respect for human rights, and more generally the stimulation of critical thinking. In authoritarian regimes, this bias may reflect values that better sustain the regime's power continuity. It is, therefore, important that developers and deployers of AIED acknowledge this responsibility and strive to ensure that educational applications - not only in terms of their content but also in terms of their teaching method - reflect a bias towards the values of democracy, human rights and the rule of law.

\subsection{Accountability}

The final requirement for Trustworthy AI - accountability - can be seen as a reiteration of all the requirements above, coupled with a demand for guarantees that these are not only proclaimed but also implemented. It entails the need for human beings to take responsibility for the outcomes and consequences of the AI-systems they develop and deploy, during the system's entire life cycle, and that they can be held accountable when adverse impacts ensue. An important aspect to enable accountability is the AIED system's auditability. A system is auditable when its design process, algorithms and data can be assessed and evaluated, not only by internal auditors but also by external auditors or other third parties. Given the importance of the role of education in societies, independent oversight of the auditing process - for instance through public entities or approved third party auditors - is not an unnecessary luxury.

The outcomes of these audits could subsequently be made public to enhance the trustworthiness both of the system and of the processes surrounding it, including the human decisions regarding its use. These evaluation reports can also indicate the (effectiveness of) measures taken to minimise unfair bias, to safeguard the system's technical robustness and safety, to render the decision-making processes more transparent, and to ensure adequate human oversight. Closely linked to this requirement is also the assessment and reporting of potential negative impacts of the AIED application, and the measures taken to address these. Such assessment needs to be tailored to the educational context in which the AI system is used, to the type of users (students, teachers, others) and must stand in proportion to the risks posed. Moreover, if the AIED system is actually deployed, the assessment needs to be carried out periodically, so as to allow a continuous evaluation.

An element that often lacks sufficient attention when ensuring accountability for AIED, concerns the importance of a protective framework for those who report on adverse impacts thereof - be it an unfair bias, a safety issue, an unfounded claim of effectiveness or another deficiency - in situations where such reporting could go against the interests of the school or of other stakeholders, or more generally raise difficulties. Whistle-blowers have already played a crucial role in shedding light on some of the problematic uses of $\mathrm{AI}$ in other contexts, and thereby contributed in rendering the developers and deployers of AI more accountable (Crawford et al. 2019). In the context of education, it is important that students, teachers, parents, employees of AIED-developing companies and other actors feel comfortable to report their concerns on an application's potential negative impacts, without a fear for potential repercussions in whatever form.

Ensuring accountability for AIED also entails the acknowledgment that tensions may arise between the above-mentioned requirements. These requirements can be implemented in multiple ways, and will in some situations necessitate balancing-acts and trade-offs. It is important that these trade-offs are not only acknowledged and documented, but also assessed in a rational and methodological manner, examining the various interests involved, as well as the consequences of the design and implementation choices for those interests. In this manner, the accountability of the entity making the trade-off can be ensured. When no ethically acceptable trade-off might exist, the AIED application should not be 
developed or used in that form, and either necessitates an overhaul of its design and purpose, or the consideration of alternative tools to reach the aspired goals. Finally, it is crucial that, whenever the development or use of AIED leads to an unjust harm - whether for students, teachers or any other stakeholder - redress can easily be sought. Those responsible - for instance the educational institution deploying AIED - should clearly communicate about the availability of redress mechanisms.

\subsection{Additional considerations for a Trustworthy AIED assessment}

In addition to the seven requirements set out above, here below some further considerations are listed for developers and deployers of AIED applications to keep in mind in the context of a contextual 'Trustworthy AIED' assessment.

First, while the Ethics Guidelines for Trustworthy AI do not explicitly contain a 'requirement zero', it has been argued that such a requirement should nevertheless be considered when contemplating the use of AI systems in sensitive contexts (Muller 2020). Prior to implementing an educational AI application, and especially when this application can have an impact on students' or teachers' lives, it is worth asking whether the problem that needs to be solved, or the task that requires a more effective execution, actually necessitates an AI-based solution. In some instances, it may be more beneficial to instead keep relying on human intervention only, or to rely on other (technological) tools that provide adequate results.

This consideration can, firstly, be made from an ethical risk-benefit perspective. Even when the use of the AI application as such can be done legally, ethically and robustly, it should still be considered whether the potential negative impact the application might have is proportionate to the increase in benefits. Secondly, this consideration can also be made from an economic cost-benefit approach. In certain situations, developing an AI system that accurately performs a task might be very costly due to the difficulty to gather the necessary data, or due to the significance of the amount of data that needs to be gathered and curated - which may not weigh up against the benefits that the system could provide as compared to other tools. Although drawn from a different sector, the example of COMPAS - a predictive AI system assessing the likelihood of recidivism based on the analysis of 137 information points about an individual - can serve as an illustration. Besides struggling with issues of bias, studies showed that a similar predictive accuracy could be achieved by using only seven information points instead of 137, and by relying either on a simple linear predictor or even on (non-expert) human beings (Dressel and Farid 2018).

This example is also relevant when designing an AIED application that, for instance, is meant to evaluate certain features of students or teachers. If the job can be done just as well with a much simpler algorithm or with far less (personal) data - and less risks - an alternative should be considered. In other words, it is not because an AIED application can be built, that it also should be built. Furthermore, there are tasks for which no AI model is easily developed, for instance due to a lack of appropriate metrics based on which a model can be created. AI is not a solution to every problem. For differing reasons, other tools can sometimes be more suited to fulfil a given task. This also raises the need to appraise the effectiveness and added value of a given AIED application more generally, which - just like for other EdTech solutions - is not always easy to do (Livingstone 2012; Selwyn 2013). Importantly, when prospective deployers of AIED applications assess costs, benefits and alternative options, they should rely not only on the expertise of the company developing or selling AIED, but on other expert opinions too.

Second, AIED applications can be based on different AI-techniques. There is no such thing as "one" AI, and applications based on deep learning will present different risks than applications based on rule- 
based AI. In the same vein, not all uses of AI within the educational sector raise the same type of issues. More vigilance will be needed for AIED applications that directly interact with or have an impact on students or teachers, as opposed to applications that merely help reduce administrative backlogs or optimise logistical aspects in a school's organisation. It also matters if AI is used in public education where no easy opt-out may be available - or in voluntary educational programmes. In sum, the various situations in which AIED is used should be differentiated from each other, so that a case-by-case assessment can be made of the measures needed to safeguard the requirements for Trustworthy AI.

Third, no uniform definition of AI exists, which may further complicate matters for those involved (Buiten 2019). The current hype around AI - and the investments focused thereon - has even led some companies to falsely claim the use of AI, in the hope that this would attract new funds (Kelnar 2019). Another definitional complication is that the boundaries of applications considered as "intelligent" are continuously changing in view of the so-called AI effect (McCorduck 2004). This definitional question should however not stand in the way of securing a proper assessment of the ethical issues that an AIED application might raise. All the requirements for Trustworthy AI can be equally applicable to automated decision-making systems that may not uniformly be considered as "AI". Rather than fixating on AI definitions, a thorough mapping should be made of the potential risks posed by the specific application and of how these risks can be mitigated - regardless of whether it concerns a simple, advanced or professed AI system.

Finally, such a risk assessment should be contextual and - for each risk identified - take into account factors such as the extent, likelihood, ubiquity and reversibility thereof. A contextual risk assessment also requires attention to the level of maturity of the individuals involved. Indeed, children have different levels of comprehension, which is a relevant factor when assessing the manner in which requirements such as transparency, explainability or human agency should be implemented in AIED applications. In this regard, inspiration can also be drawn from the ample literature that already exists on the impact of technology and the internet more generally on children. Important research on this has, for instance, been carried out from a sociological and educational perspective (such as, for instance, Livingstone 2011; Kardefelt-Winther 2017; Livingstone, Haddon, and Görzig 2012), which can be a basis for further reflection in the context of AIED-specific assessments.

\section{Pathways to secure Trustworthy AIED}

Despite the slow start, the debate on ethical AI is finally reaching the educational domain. This was at least in part - spurred by the COVID-19 pandemic, which led to a fast-paced adoption of digital and AI-based educational solutions across the world, not always in a manner that aligned with ethical values. Indeed, the deployment of certain AIED applications sparked a public outrage precisely because the adequate assessment and mitigation of ethical risks was lacking. The increased attention for ethical AIED is a welcome development. It not only enriches the public debate, but also enhances the awareness of those directly involved that AIED can come with certain risks for which responsibility needs to be taken. It also sends a signal to regulators that some of these risks may require regulatory responses that go beyond non-binding guidance.

In the sections above, an overview was provided of the seven requirements for Trustworthy AI, and the peculiar challenges faced by the educational sector to ensure their implementation. While the Guidelines - and in particular, the assessment list that helps operationalise the seven requirements can provide a useful tool for AIED developers and deployers, the question however remains: are horizontal guidelines sufficient? Even if they are duly implemented in a manner that is tailored to the educational realm, does a voluntary approach to comply with these requirements suffice? If not, what is the way forward to ensure that the ethical risks raised by AIED are adequately tackled, and that those 
involved can be held accountable when this does not occur? An appropriate balance should be found between - on the one hand - taking courage from the fact that organisations are increasingly aware of AI's ethical issues and are starting to take measures to mitigate them, and - on the other hand - being naive in thinking that commercial interests are fully aligned with the interest of those that can be negatively impacted. Besides disseminating and encouraging adherence to non-binding guidance, a number of alternative and complementary pathways are open to policymakers who - given the extent of some of the risks posed by AIED - have a responsibility to ensure their careful consideration.

To start with, they should acknowledge that the increasing ubiquity of AI necessitates a broader awareness and understanding of this technology by society at large. Education can not only be an application domain for AI systems, but can - and should - also be used as an enabler to foster knowledge on this technology and ensure that the benefits, challenges and limitations thereof are duly explained. Digital literacy classes can serve this aim, but should not be restricted to the development of ICT skills. Rather, they should also encompass education on digital rights (such as privacy and data protection, but also freedom of speech and thought), digital communication, digital safety and security, digital identity, and other capabilities that are needed for a holistic picture of AI systems. For this purpose, work is also needed to educate the educators, and ensure that teachers are able to address these topics with their students in a critical manner. At the very least, the use of AIED in classes should be coupled with lessons on how AI works and in which ways it is limited. While an updated curriculum is not the panacea to counter the ethical risks raised by AIED, it is both useful and essential.

Second, policy-makers should carefully reflect upon the role of commercial actors in the educational sphere. This debate is not new, as many EdTech solutions - including those using AI techniques - are developed by private companies and are hence at least partially guided by commercial interests. Those companies - and the beneficial AIED innovations they may generate - can make important contributions to the quality and availability of education. At the same time, national governments and educational institutions alike depend on these private actors when seeking to procure or deploy AIED. Given the ethical challenges set out above, and the impact that some of these applications can have both on the educational process and on people's lives and minds, it should be ensured that the commercial nature of AIED applications does not interfere with the rights and interests of those involved, but rather empowers them. To this end, policy-makers adopting strategies on technology and education should foster a broader reflection on how this can be ensured (Selwyn 2016). As noted above, such reflection should go beyond considerations of cybersecurity and data protection (to which the UK's strategy, for instance, currently seems limited (UK Department for Education 2019)).

Third, for certain AIED applications, more far-going measures may be needed. For instance, the invasiveness of applications that entail physical or mental student tracking through biometric recognition techniques renders them an important candidate for mandatory regulatory oversight. AI applications that run on such data, including emotional tracking, behavioural identification, affect recognition, voice and tone recognition, facial recognition and the analysis of micro-expressions, constitute a significant intrusion of individuals privacy (McStay 2019). Not only are many of the applications that gather such data unable to deliver their promised benefits, they are also prone to erroneous judgments and bias, which can negatively impact those who are subjected thereto. However, also for other AIED applications that can have an impact on the rights of students or teachers - such as the right to non-discrimination - enhanced oversight may be warranted.

As noted above, a number of regulators are currently considering the adoption of new regulation for AI applications. Given the prominence of power asymmetries in the educational realm, and given the prevalent involvement of vulnerable individuals, it is important that regulators acknowledge the educational sector as meriting heightened scrutiny. While many AIED applications can undoubtedly 
benefit the stakeholders involved and not all such applications raise the same level of risks, for all the reasons set out above, the educational realm should be included in any 'high-risk sectors' list. New legal requirements that may befall 'high-risk' AI-systems under upcoming legislative proposals should also apply to high-risk AI systems in the education sector.

Across the globe, including in Europe, many schools are still facing difficulties to secure basic educational IT infrastructure, such as an internet connection and properly functioning soft- and hardware. Amongst and within countries, strong inequalities exist between the level of education that students enjoy and the digital literacy they are taught. While AIED promises to make education more broadly accessible and hence can help reduce these inequalities, it can just as well exacerbate them and widen the digital literacy gap. At the same time, data is increasingly used as a currency, traded off against services claiming to be free. There is hence a danger that schools unable to afford AIED applications will instead be encouraged to use the currency of (their students') data as a commodity in exchange for the technology's promised benefits, without any mapping of the potential adverse impacts, without ethical safeguards, and without any sound evidence that the claimed benefits are worth the risks. In this context, consent must hence be treated with extreme care when used as a legal basis for the processing of (sensitive) data and additional safeguards will be needed to ensure not only respect for privacy, but also for all the other requirements of Trustworthy AI.

Such safeguards can for instance consist of mandatory documentation obligations for certain AIED applications. Besides enhancing the traceability - and hence auditability - of these applications, such documentation could also describe the methodology that was used to secure the systems' technical robustness, explain the design choices made, and describe the implemented testing and verification procedures to tackle unfair bias and other risks. Public authorities - and relevant independent third parties such as NGOs - should be able to audit these systems to assess whether they match their documentation and whether they abide by applicable laws. In addition, when AIED applications are acquired through tender procedures or public procurement processes, governments should demand a comprehensive assessment of the system's ethical, social and societal impact.

Complementary to the above pathways, the development of tailored guidelines for the use of AI in education would not be a luxury given the specific ethical issues raised by AIED. Furthermore, besides more tailored guidelines and legal safeguards, the development of certification procedures specifically for AIED can also be of help, as it provides AIED developers with a tool to not only claim but also demonstrate compliance. It should, however, be stressed that any regulation, domain-specific guidelines and certification mechanisms ought to be developed in a multi-stakeholder and multi-disciplinary setting, bringing together AIED developers and data scientists, pedagogists, teachers, ethicists, psychologists and other experts, but ideally also students or student representatives.

Given that research on the impact of AI's mid- and longer-term consequences is lacking, a risk-based approach is warranted, which duly takes into consideration the essential role of education in our society, as well as in the lives of the 2.2 billion of children around the world. If for every euro of research that goes into the development and analysis of the potential benefits of AIED a euro can also be spent on research into the ethical risks thereof and the measures that can help addressing these, the goal of attaining Trustworthy AIED will come a little closer.

\section{Conclusions}

Artificial Intelligence is altering all domains of our lives, from the way we communicate and work to the way we behave and think. Like virtually all technologies, it can be used for beneficial purposes, it can be used for harmful purposes, and it can be used in ways that cause harm in entirely unintentional 
ways. The extent of this harm is strongly dependent on the application in question and the context in which it is used, as well as on those directly or indirectly involved. The question should hence be: whom does the claimed beneficial purpose of AI systems serve, and who might be impacted in potentially harmful ways? While AI applications and ed-tech more broadly have the potential to foster higher quality education across the globe and hence contribute to the fourth Sustainable Development Goal, it can also exacerbate existing inequalities and discrimination, widen the digital literacy gap, infringe on individuals' privacy, inadvertently influence or manipulate its users and pose other significant risks in a democratic society. The particular role of education in our societies also raises a number of domainspecific sensitivities for the development and use of AI in the educational context. This particular role - and the opportunities and risks it entails - is exemplified by the quotes of two notorious historical figures, one of them is considered as one of the morally most reprehensible individuals of the last century, the other as one of the most morally valued:

- Whoever has the youth has the future - A. Hitler

- Education is the most powerful weapon which you can use to change the world - N. Mandela

Both have recognised the importance of education as a tool to shape society, and both have striven to embed their ideals in the educational systems of their respective jurisdictions. It is a collective responsibility to safeguard the ideals of a liberal and democratic society, and to make sure that AI used in the domain of education reflects these ideals, both as regards the educational content it provides and the manner in which it operates. Notwithstanding sensational media reporting about the subject, AI is not something that suddenly overcomes us. While undoubtedly being a powerful technology, it remains up to human beings to decide how and for which purposes it is developed, when and where it can be deployed, and under which conditions and safeguards it should be used. This responsibility is particularly important when those involved do not (yet) have a strong voice of their own, either because of their young age or because of their more vulnerable position in society.

While it is necessary to distinguish between the different applications of AIED - which certainly do not all present the same type and extent of risk - a public debate needs to be held, in an informed manner and with the involvement all relevant stakeholders, to collectively decide how we wish to deal with these risks. Foregoing such a debate risks leading us to either of two undesired paths: a lack of trust in the technology, leading to a technological backlash and missing out the benefits that Trustworthy AIED can bring; or the proliferation of untrustworthy AIED applications that fail to take into account the risks they pose and can lead to irreparable harm.

Adherence to ethical guidelines such as those drafted by the HLEG on AI is a necessary step to secure the goal of Trustworthy AIED, but a first step only. These guidelines can help stimulate reflection on the choices made throughout AIED's lifecycle, and stress the non-negligible fact that these choices have ethical consequences. However, they are voluntary, the overview of ethical issues they provide is nonexhaustive, and they still need to be tailored to the educational context and to the specific AI application that is envisaged. Moreover, the invasiveness of certain AIED applications render even the development of education-specific ethics guidelines an insufficient safeguard. Instead, high-risk AIED applications should be able to demonstrate their legal, ethical and robust nature. For these applications, policies should hence be established to secure mandatory safeguards, be it through documentation obligations, auditability requirements or the creation of sectorial certification mechanisms - or a combination of all.

Ultimately, however noteworthy the benefits of AIED might be, they will not be able to fulfil the purpose of enhancing learners' capabilities without taking into account not only their educational, but also their moral, mental and physical wellbeing. The various effects of the use of AI in the educational context - in particular when minors are involved - are still not fully mapped. Accordingly, further research on this subject should be fostered, as well as the mapping of appropriate technical and non- 
technical tools to help address any negative effects. At the same time, the increased attention to ethical $\mathrm{AI}$ in the educational sector allows for cautious optimism. Out of the increasingly loud calls for ethical AI, a path towards building Trustworthy AIED is slowly emerging. To truly reap the benefits thereof not only in the short but also in the longer term - all stakeholders have a responsibility to ensure it stays on track.

\section{Acknowledgments}

The author is grateful for the helpful feedback she received from Gry Hasselbalch on an earlier version of this draft.

\section{Bibliography}

ACLU. 2020. 'ACLU Civil Rights Concerns with Potential Use of Facial Recognition in Proctoring the California Bar $\quad$ Examination', $16 \quad$ July 2020. https://www.aclunc.org/sites/default/files/ACLU_Advocacy_Letter_re_Online_Bar_Exam.pd f.

Afifi-Sabet, Keumars. 2020. 'UK Gov's “Unfair” A-Level Algorithm Faces Several Legal Challenges'. IT Pro, August 2020.

Aiken, Robert M, and Richard G Epstein. 2000. 'Ethical Guidelines for AI in Education: Starting a Conversation'. International Journal of Artificial Intelligence in Education 11 (January): 16376.

Ala-Pietilä, Pekka, and Nathalie A. Smuha. 2020. 'A Framework for Global Cooperation on Artificial Intelligence and Its Governance'. In Forthcoming in 'Reflections of AI for Humanity', Springer (2021), edited by B. Braunschweig and M. Ghallab. https://ssrn.com/abstract=3696519.

Amrein-Beardsley, Audrey. 2014. Rethinking Value-Added Models in Education: Critical Perspectives on Tests and Assessment-Based Accountability. 1st Edition. New York: Routledge.

Andrea Kulkarni. 2019. 'AI in Education: Where Is It Now and What Is the Future?' Lexalytics. 6 September 2019. https://www.lexalytics.com/lexablog/ai-in-education-present-future-ethics.

Banaji, Mahzarin R, Curtis Hardin, and Alexander J Rothman. 1993. 'Implicit Stereotyping in Person Judgment'. Journal of Personality and Social Psychology 65 (2): 10.

Barrett, Lisa Feldman, Ralph Adolphs, Stacy Marsella, Aleix M. Martinez, and Seth D. Pollak. 2019. 'Emotional Expressions Reconsidered: Challenges to Inferring Emotion From Human Facial Movements'. Psychological Science in the Public Interest 20 (1): 1-68. https://doi.org/10.1177/1529100619832930.

Bedingfield, Will. 2020. 'Everything That Went Wrong with the Botched A-Levels Algorithm'. Wired UK, 19 August 2020. https://www.wired.co.uk/article/alevel-exam-algorithm.

Begby, Endre. 2013. 'The Epistemology of Prejudice'. Thought: A Journal of Philosophy 2 (2): 90-99. https://doi.org/10.1002/tht3.71.

Benjamin, Ruha. 2019. Race After Technology: Abolitionist Tools for the New Jim Code. 1 edition. Medford, MA: Polity.

Bjørnsten, Thomas Bøgevald, and Mette-Marie Zacher Sørensen. 2017. 'Uncertainties of Facial Emotion Recognition Technologies and the Automation of Emotional Labour'. Digital Creativity 28 (4): 297-307. https://doi.org/10.1080/14626268.2017.1383271.

Brkan, M. 2019. 'Artificial Intelligence and Democracy': Delphi - Interdisciplinary Review of Emerging Technologies 2 (2): 66-71. https://doi.org/10.21552/delphi/2019/2/4.

Bryson, Joanna. 2020. 'It's (Still) Not about Trust: No One Should Buy AI If Governments Won't Prosecute Liability'. It's (Still) Not about Trust (blog). 23 September 2020. https://joannabryson.blogspot.com/2020/09/its-still-not-about-trust-no-one-should.html.

Buiten, Miriam C. 2019. 'Towards Intelligent Regulation of Artificial Intelligence'. European Journal of Risk Regulation 10 (1): 41-59. https://doi.org/10.1017/err.2019.8. 
Bulger, Monica. 2016. 'Personalized Learning: The Conversations We're Not Having'. Data \& Society, July, 29.

Bulger, Monica, Patrick McCormick, and Mikaela Pitcan. 2017. 'The Legacy of InBloom', 34.

CEPEJ. 2018. 'European Ethical Charter on the Use of Artificial Intelligence in Judicial Systems and Their Environment'. Adopted at the 31st plenary meeting of the CEPEJ in Strasbourg. https://rm.coe.int/ethical-charter-en-for-publication-4-december-2018/16808f699c.

Coughlan, Sean. 2020. 'Why Did the A-Level Algorithm Say No?' BBC News, 14 August 2020, sec. Family \& Education. https://www.bbc.com/news/education-53787203.

Council of Europe. 2019. 'Terms of Reference for the Ad Hoc Committee on Artificial Intelligence (CAHAI)'.

Crawford, Kate, Roel Dobbe, Theodora Dryer, Genevieve Fried, Ben Green, Elizabeth Kaziunas, Amba Kak, et al. 2019. AI Now 2019 Report. New York: AI Now Institute. https://ainowinstitute.org/AI_Now_2019_Report.pdf.

Dressel, Julia, and Hany Farid. 2018. 'The Accuracy, Fairness, and Limits of Predicting Recidivism'. Science Advances 4 (1): eaao5580. https://doi.org/10.1126/sciadv.aao5580.

European Commission. 2018. 'Artificial Intelligence for Europe'. $\operatorname{COM}(2018) 237$ final. https://ec.europa.eu/newsroom/dae/document.cfm?doc_id=51625.

. 2019. 'Building Trust in Human-Centric Artificial Intelligence'. COM(2019) 168 final. European Commission. https://ec.europa.eu/newsroom/dae/document.cfm?doc_id=58496. 2020. 'White Paper On Artificial Intelligence - A European Approach to Excellence and Trust'. Brussels, 19.2.2020 COM(2020) 65 final.

Francesc, Pedró, Miguel Subosa, Axel Rivas, and Paula Valverde. 2019. 'Artificial Intelligence in Education: Challenges and Opportunities for Sustainable Development'. UNESCO. https://unesdoc.unesco.org/ark:/48223/pf0000366994.

Gallo, David. 2015. 'La politique de formation idéologique de la SS (1933-1945). Une étude sur la transmission de la normativité nazie'. Revue historique 676 (4): 875. https://doi.org/10.3917/rhis.154.0875.

Guo, Anhong, Ece Kamar, Jennifer Wortman Vaughan, Hanna Wallach, and Meredith Ringel Morris. 2019. 'Toward Fairness in AI for People with Disabilities: A Research Roadmap'. ACM ASSETS, 2019 Workshop on AI Fairness for People with Disabilities, , 9.

Han, Hye Jung. 2020. 'An Algorithm Shouldn't Decide a Student's Future'. Politico, 13 August 2020. https://www.politico.eu/article/an-algorithm-shouldnt-decide-students-future-coronavirusinternational-baccalaureate/.

Hanselaer, Sarah. 2019. 'Facial recognition in school renders Sweden's first GDPR fine'. Text. European Data Protection Board - European Data Protection Board. 22 August 2019. https://edpb.europa.eu/news/national-news/2019/facial-recognition-school-renders-swedensfirst-gdpr-fine_en.

Hao, Karen. 2019. 'Training a Single AI Model Can Emit as Much Carbon as Five Cars in Their Lifetimes'. MIT Technology Review (blog). 6 June 2019. https://www.technologyreview.com/2019/06/06/239031/training-a-single-ai-model-can-emitas-much-carbon-as-five-cars-in-their-lifetimes/.

Harwell, Drew. 2019. 'A Face-Scanning Algorithm Increasingly Decides Whether You Deserve the Job'. Washington Post, 6019. https://www.washingtonpost.com/technology/2019/10/22/ai-hiring-face-scanning-algorithmincreasingly-decides-whether-you-deserve-job/.

Hasselbalch, Gry. 2019. 'Making Sense of Data Ethics. The Powers behind the Data Ethics Debate in European Policymaking'. Internet Policy Review 8 (2). https://doi.org/10.14763/2019.2.1401.

Herold, Benjamin. 2018. 'Ed-Tech Companies Tracking Students' Emotions, Mindsets: A Push to Use New Technology to Understand the "whole Child" Is Sparking Privacy Fears'. Education Week; $\quad$ Bethesda 37 http://search.proquest.com/docview/2058514954?rfr_id=info\%3Axri\%2Fsid\%3Aprimo.

(36).

Hibbeln, Martin, Jeffrey Jenkins, Christoph Schneider, Joseph Valacich, and Markus Weinmann. 2017. 'How Is Your User Feeling? Inferring Emotion Through Human-Computer Interaction Devices'. MIS Quarterly 41 (1): 1-21. https://doi.org/10.25300/MISQ/2017/41.1.01. 
High-Level Expert Group on AI. 2019a. 'A Definition of AI: Main Capabilities and Scientific Disciplines'. . 2019b. 'Ethics Guidelines for Trustworthy AI'.

Isaak, Jim, and Mina J. Hanna. 2018. 'User Data Privacy: Facebook, Cambridge Analytica, and Privacy Protection'. Computer 51 (8): 56-59. https://doi.org/10.1109/MC.2018.3191268.

Jobin, Anna, Marcello Ienca, and Effy Vayena. 2019. 'The Global Landscape of AI Ethics Guidelines'. Nat Mach Intell 1: 389-399.

Johar, Swati. 2016. Emotion, Affect and Personality in Speech. SpringerBriefs in Electrical and Computer Engineering. Cham: Springer International Publishing. https://doi.org/10.1007/9783-319-28047-9.

Johnson, Khari. 2020. 'ExamSoft's Remote Bar Exam Sparks Privacy and Facial Recognition Concerns'. $\quad$ VentureBeat $\quad$ (blog). $29 \quad 2020$. https://venturebeat.com/2020/09/29/examsofts-remote-bar-exam-sparks-privacy-and-facialrecognition-concerns/.

Kardefelt-Winther, Daniel. 2017. 'How Does the Time Children Spend Using Digital Technology Impact Their Mental Well-Being, Social Relationships and Physical Activity?: An EvidenceFocused Literature Review'. Innocenti Discussion Papers 2017/02. Vol. 2017/02. Innocenti Discussion Papers. UNICEF. https://doi.org/10.18356/cfa6bcbl-en.

Kelnar, David. 2019. 'The State of AI 2019: Divergence'. MMC Ventures. https://www.mmcventures.com/wp-content/uploads/2019/02/The-State-of-AI-2019Divergence.pdf.

Koenecke, Allison, Andrew Nam, Emily Lake, Joe Nudell, Minnie Quartey, Zion Mengesha, Connor Toups, John R. Rickford, Dan Jurafsky, and Sharad Goel. 2020. 'Racial Disparities in Automated Speech Recognition'. Proceedings of the National Academy of Sciences 117 (14): 7684-89. https://doi.org/10.1073/pnas.1915768117.

Kunzer, Edward J. 1938. 'The Youth of Nazi Germany'. Journal of Educational Sociology 11 (6): 342. https://doi.org/10.2307/2262246.

Leenes, Ronald, Erica Palmerini, Bert-Jaap Koops, Andrea Bertolini, Pericle Salvini, and Federica Lucivero. 2017. 'Regulatory Challenges of Robotics: Some Guidelines for Addressing Legal and Ethical Issues'. Law, Innovation and Technology 9 (1): 1-44. https://doi.org/10.1080/17579961.2017.1304921.

Lieberman, Mark. 2018. 'Sentiment Analysis Allows Instructors to Shape Course Content around Students' Emotions'. Inside Higher Ed. 20 February 2018. https://www.insidehighered.com/digital-learning/article/2018/02/20/sentiment-analysisallows-instructors-shape-course-content.

Lin, Patrick, Keith Abney, and George A. Bekey, eds. 2014. Robot Ethics - The Ethical and Social Implications of Robotics. Cambridge, Mass: MIT Press. https://mitpress.mit.edu/books/robotethics.

Livingstone, Sonia. 2011. 'Internet, Children, and Youth'. In The Handbook of Internet Studies, edited by Mia Consalvo and Charles Ess, 348-68. Oxford, UK: Wiley-Blackwell. https://doi.org/10.1002/9781444314861.ch16.

- 2012. 'Critical Reflections on the Benefits of ICT in Education'. Oxford Review of Education 38 (1): 9-24. https://doi.org/10.1080/03054985.2011.577938.

Livingstone, Sonia M., Leslie Haddon, and Anke Görzig, eds. 2012. Children, Risk and Safety on the Internet: Research and Policy Challenges in Comparative Perspective. Bristol: Policy Press.

McCorduck, Pamela. 2004. Machines Who Think. 2nd ed. Natick, MA: A. K. Peters, Ltd.

McKinsey. 2018. 'Notes from the AI Frontier: Modeling the Impact of AI on the World Economy'. Discussion Paper. McKinsey. https://www.mckinsey.com/ /media/McKinsey/Featured\%20Insights/Artificial\%20Intelligen ce/Notes $\% 20$ from $\% 20$ the $\% 20$ frontier $\% 20$ Modeling $\% 20$ the $\% 20$ impact $\% 20$ of $\% 20 \mathrm{AI} \% 20 \mathrm{on} \%$ 20the\%20world\%20economy/MGI-Notes-from-the-AI-frontier-Modeling-the-impact-of-AIon-the-world-economy-September-2018.pdf.

McStay, Andrew. 2019. 'Emotional AI and EdTech: Serving the Public Good?' Learning, Media and Technology, November, 1-14. https://doi.org/10.1080/17439884.2020.1686016. 
Mittelstadt, Brent Daniel, Patrick Allo, Mariarosaria Taddeo, Sandra Wachter, and Luciano Floridi. 2016. 'The Ethics of Algorithms: Mapping the Debate'. Big Data \& Society 3 (2): 205395171667967. https://doi.org/10.1177/2053951716679679.

Monetary Authority of Singapore. 2018. 'Principles to Promote Fairness, Ethics, Accountability and Transparency (FEAT) in the Use of Artificial Intelligence and Data Analytics in Singapore's Financial Sector'. https://www.mas.gov.sg/ /media/MAS/News\%20and\%20Publications/Monographs\%20and \%20Information\%20Papers/FEAT\%20Principles\%20Final.pdf.

Muller, Catelijne. 2020. 'The Impact of Artificial Intelligence on Human Rights, Democracy and the Rule of Law'. CAHAI(2020)06-fin. Report Prepared in the Context of the Council of Europe's Ad Hoc Committee on AI (CAHAI). Strasbourg: Council of Europe. https://www.coe.int/en/web/artificial-intelligence/cahai.

Ntoutsi, Eirini, Pavlos Fafalios, Ujwal Gadiraju, Vasileios Iosifidis, Wolfgang Nejdl, Maria-Esther Vidal, Salvatore Ruggieri, et al. 2020. 'Bias in Data-Driven Artificial Intelligence SystemsAn Introductory Survey'. WIREs Data Mining and Knowledge Discovery 10 (3): e1356. https://doi.org/10.1002/widm.1356.

O’Neil, Cathy. 2017. Weapons of Math Destruction. Penguin Books Ltd.

Poel, Ibo van de, and Peter Kroes. 2014. 'Can Technology Embody Values?' In The Moral Status of Technical Artefacts, edited by Peter Kroes and Peter-Paul Verbeek, 17:103-24. Dordrecht: Springer Netherlands. https://doi.org/10.1007/978-94-007-7914-3_7.

Prinsloo, Paul, and Sharon Slade. 2016. 'Student Vulnerability, Agency and Learning Analytics: An Exploration'. Journal of Learning Analytics 3 (1): 159-182-159-182. https://doi.org/10.18608/jla.2016.31.10.

Raza, Zainab. 2019. 'CHINA'S "POLITICAL RE-EDUCATION" CAMPS OF XINJIANG'S UYGHUR MUSLIMS'. Asian Affairs 50 (4): 488-501. https://doi.org/10.1080/03068374.2019.1672433.

Rouhiainen, Lasse. 2019. 'How AI and Data Could Personalize Higher Education'. Harvard Business Review, 14 October 2019. https://hbr.org/2019/10/how-ai-and-data-could-personalize-highereducation.

Schwartz, Roy, Jesse Dodge, Noah A. Smith, and Oren Etzioni. 2019. 'Green AI'. ArXiv:1907.10597 [Cs, Stat], August. http://arxiv.org/abs/1907.10597.

Selwyn, Neil. 2013. Distrusting Educational Technology: Critical Questions for Changing Times. New York: Routledge. https://doi.org/10.4324/9781315886350.

2016. Is Technology Good for Education? Cambridge: Polity Press. http://limo.libis.be/primoexplore/fulldisplay/32LIBIS_ALMA_DS71200759640001471/KULeuven.

Silberg, Jake, and James Manyika. 2019. 'Notes from the AI Frontier - Tackling Bias in AI (and in Humans)'. McKinsey Global Institute. https://www.mckinsey.com/ /media/McKinsey/Featured\%20Insights/Artificial\%20Intelligen ce/Tackling\%20bias\%20in\%20artificial\%20intelligence\%20and\%20in\%20humans/MGITackling-bias-in-AI-June-2019.pdf.

Smuha, Nathalie A. 2019. 'The EU Approach to Ethics Guidelines for Trustworthy Artificial Intelligence'. Computer Law Review International 20 (4): 97-106.

Southgate, Erica, Karen Blackmore, Stephanie Pieschl, Susan Grimes, Jessey McGuire, and Kate Smithers. 2018. 'Artificial Intelligence and Emerging Technologies in Schools'. Newcastle: University of Newcastle, Australia: Australian Government Department of Education.

Stark, Luke. 2018. 'Algorithmic Psychometrics and the Scalable Subject'. Social Studies of Science 48 (2): 204-31. https://doi.org/10.1177/0306312718772094.

Steinbock, Bonnie. 1978. 'Speciesism and the Idea of Equality'. Philosophy 53 (204): 247-56.

Strubell, Emma, Ananya Ganesh, and Andrew McCallum. 2019. 'Energy and Policy Considerations for Deep Learning in NLP'. ArXiv:1906.02243 [Cs], June. http://arxiv.org/abs/1906.02243.

Tschopp, Marisa. 2020. 'Digital Transformation - Three Wrong Questions about Trust and AI'. DigitalCommerce - $\quad$ Die Post. 23 September 2020. https://digitalcommerce.post.ch/en/pages/blog/2020/trust-in-artificial-intelligence.

Turing, Alan. 1950. 'Computing Machinery and Intelligence'. Mind 59 (236): 433-60. 
UK Department for Education. 2019. 'Realising the Potential of Technology in Education: A Strategy for Education Providers and the Technology Industry'. https://assets.publishing.service.gov.uk/government/uploads/system/uploads/attachment_data/ file/791931/DfE-Education_Technology_Strategy.pdf.

UK Department of Health and Social Care. 2019. 'Code of Conduct for Data-Driven Health and Care Technology'. https://www.gov.uk/government/publications/code-of-conduct-for-data-drivenhealth-and-care-technology/initial-code-of-conduct-for-data-driven-health-and-caretechnology.

UNESCO. 2019. 'Beijing Consensus on Artificial Intelligence and Education'. Outcome document of the International Conference on Artificial Intelligence and Education 'Planning education in the AI era: Lead the leap'. Beijing: UNESCO. https://unesdoc.unesco.org/ark:/48223/pf0000368303.

UNICEF. 2020. 'Policy Guidance on AI for Children (Draft)'. https://www.unicef.org/globalinsight/media/1171/file/UNICEF-Global-Insight-policyguidance-AI-children-draft-1.0-2020.pdf.

United Nations. 2006. United Nations Convention on the Rights of Persons with Disabilities. https://reaties.un.org/Pages/ViewDetails.aspx?src=TREATY\&mtdsg_no=IV$15 \&$ chapter $=4 \&$ clang $=$ en.

Vincent-Lancrin, Stéphan. 2020. 'Trustworthy Artificial Intelligence (AI) in Education: Promises and Challenges'. OECD Education Working Paper No. 218. OECD, Directorate for Education and Skills.

Vinuesa, Ricardo, Hossein Azizpour, Iolanda Leite, Madeline Balaam, Virginia Dignum, Sami Domisch, Anna Felländer, Simone Daniela Langhans, Max Tegmark, and Francesco Fuso Nerini. 2020. 'The Role of Artificial Intelligence in Achieving the Sustainable Development Goals'. Nature Communications 11 (1): 233. https://doi.org/10.1038/s41467-019-14108-y.

Yeung, Karen. 2019. 'Why Worry about Decision-Making by Machine?' In Algorithmic Regulation, edited by Karen Yeung and Martin Lodge, 21-48. Oxford University Press. https://doi.org/10.1093/oso/9780198838494.003.0002.

Yujie, Xue. 2019. 'Camera Above the Classroom'. Sixth Tone. 26 March 2019. https://www.sixthtone.com/news/1003759/camera-above-the-classroom.

Zenz, Adrian. 2019. “Thoroughly Reforming Them towards a Healthy Heart Attitude": China's Political Re-Education Campaign in Xinjiang'. Central Asian Survey 38 (1): 102-28. https://doi.org/10.1080/02634937.2018.1507997. 